\title{
Managing Diabetes During the COVID-19 Pandemic
}

\author{
John Doupis and Konstantinos Avramidis \\ Department of Internal Medicine and Diabetes, Salamis Naval and Veterans Hospital, Salamis Naval Base, Attiki, Greece
}

DOI: https://doi.org/10.17925/EE.2020.16.2.85

$\mathrm{T}$ he coronavirus disease 2019 (COVID-19) pandemic has affected almost every country in the world and has changed the way we access healthcare. People with pre-existing conditions, such as diabetes, are at high risk of a severe disease course and it is essential that, as well as good hygiene and social distancing measures, blood glucose is carefully monitored, as chronic hyperglycaemia can lead to immune dysfunction. People with diabetes should be encouraged to continue medication prescribed for hypertension, diabetes or dyslipidaemia. Furthermore, patients with diabetes and COVID-19 infection should follow their usual antidiabetic treatment with the exception of sodium-glucose cotransporter-2 inhibitors. As the current pandemic situation has rendered some patients unable to access routine healthcare, telehealth may help those with travel restrictions.

\section{Keywords}

COVID-19, diabetes, SARS-CoV-2, glucose control

Disclosures: John Doupis and Konstantinos Avramidis have no financial or non-financial relationships or activities to declare in relation to this article.

Review Process: Double-blind peer review.

Compliance with Ethics: This article is an opinion piece and does not report on new clinical data, or any studies with human or animal subjects performed by either of the authors.

Authorship: The named authors meet the International Committee of Medical Journal Editors (ICMJE) criteria for authorship of this manuscript, take responsibility for the integrity of the work as a whole, and have given final approval for the version to be published. Access: This article is freely accessible at touchENDOCRINOLOGY.com (c) Touch Medical Media 2020

Received: 19 May 2020

Accepted: 9 June 2020

Published Online: 2 October 2020

Citation: European Endocrinology. 2020;16(2):85-7

Corresponding Author: John Doupis,

Department of Internal Medicine and Diabetes Salamis

Naval and Veterans Hospital, Salamis Naval Base, 18900

Salamis, Attiki, Greece.

E: john.doupis@harvard-alumni.org

Support: No funding was received in the publication of this article.
The coronavirus disease 2019 (COVID-19) pandemic is an occurring global public health crisis due to the spread of the severe acute respiratory syndrome coronavirus 2 (SARS-CoV-2). ${ }^{1}$ In December of 2019, a low respiratory tract infection of unknown cause emerged in Wuhan, Hubei Province, China. The Huanan seafood market was considered to be the main source of the responsible pathogen; bats sold on this market were proposed to be the most probable origin of the virus, given that they serve as a natural reservoir for coronaviruses. ${ }^{2}$ The World Health Organization (WHO) declared the COVID-19 outbreak as a pandemic on 11 March 2020, reporting more than 120,000 cases and 4,000 deaths worldwide at this timepoint. ${ }^{3}$ The virus is mainly spread through air droplets, and the most common symptoms of the disease include fever, dry cough, fatigue, expectoration and dyspnoea, as well as taste and smell impairment. ${ }^{4}$ In some cases, myalgia, pharyngodynia, headache, nausea, nasal congestion and diarrhoea are also present. However, some patients may be asymptomatic carriers. ${ }^{4}$ As of 10 June 2020, more than 7.2 million cases of COVID-19 had been reported in over 188 countries, resulting in more than 400,000 deaths. More than 3.3 million people have recovered. ${ }^{5}$ High disease mortality and evidence of worse prognosis in patients with diabetes, urges the need for a thorough discussion regarding the effective management of diabetes during this pandemic.

\section{Outcomes in patients with COVID-19 and diabetes}

Diabetes mellitus has been associated with poor outcomes in patients with COVID-19.6-11 A meta-analysis of 6,452 patients from 30 studies proposed a close association of severe COVID-19 infection in the presence of diabetes, with a high prevalence of acute respiratory distress syndrome, fast disease progression and high mortality. This association becomes even stronger in patients with diabetes and other comorbidities like hypertension, cardiovascular disease, chronic kidney disease and severe obesity (body index mass $>40 \mathrm{~kg} / \mathrm{m}^{2}$ ). In such cases, intensive care unit (ICU) admission is more frequently required. Acute complications include acute cardiac injury, acute liver dysfunction and acute kidney failure. In addition, obese individuals on mechanical ventilation are at higher risk for ventilatory failure, as high body mass index is a major determinant of lung volume.7.8

It is commonly acknowledged that chronic hyperglycaemia may lead to immune dysfunction and a subsequent susceptibility to infections.9-11 Thus, innate immunity is an inadequate first-line barrier against SARS-CoV-2. Furthermore, an exaggerated immune response with cytokine overproduction, such as interleukin 6 (IL-6), as well as increased levels of C-reactive protein and ferritin, could possibly explain the vulnerability of diabetic patients to the cytokine storm, shock and COVID-19-related fatal complications.9-11 In addition, high D-dimer levels in patients with diabetes and COVID-19 may serve as a marker for the over-activation of the coagulation cascade, which increases mortality through major thromboembolic events. ${ }^{12}$ Last, but not least, angiotensin-converting enzyme 2 (ACE2) overexpression on various cell membranes, partly explained due to ACE2 upregulation as a result of the chronic use of ACE inhibitors, hypoglycaemic agents and statins, may have a beneficial effect; a recent study has shown that ACE inhibitor use was associated with reduced risk for hospitalisation.,13 


\section{Treating non-infected patients with diabetes}

Poor diabetes control has been proven to negatively affect prognosis and promote the risk of any infection, thus, tight glucose control is required

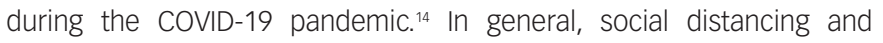
good hygiene should be the norm of an effective prevention strategy. ${ }^{15}$ Nevertheless, possible travel restrictions and quarantine should not undermine the level of necessary healthcare services that need to be provided. Thus, access to healthcare providers should be ensured during the COVID-19 pandemic. ${ }^{14}$ For this reason, telehealth services may be a useful tool for patient consultation or even early recognition of possible diabetes complications and signs of blood glucose dysregulation or infection. ${ }^{11,16}$ In addition, patients should obtain enough supplies of medication, as well as, glucose needles, strips or sensors for home use. ${ }^{14}$ Access to social care professionals may be mandatory in some cases, considering that stress management is crucial for mental and overall health balance..$^{16}$

There is no evidence supporting discontinuation of medication prescribed for hypertension, diabetes or dyslipidaemia; regular intake of antidiabetic drugs and insulin is indicated. ${ }^{11,15}$ Although many theories suggesting that chronic use of ACE inhibitors and angiotensin 2 receptor blockers (ARBS) may increase the risk and the severity of SARS-CoV-2 infection, the Council on Hypertension of the European Society of Cardiology (ESC Council) strongly recommends the continuation of the usual chronic anti-hypertensive treatment, as there is a lack of evidence for a possible harmfulness of these drugs. ${ }^{17}$ In a recent publication, clinical outcomes in COVID-19 patients with diabetes and hypertension receiving ACE inhibitors/ARBS were similar to the control group. ${ }^{18}$ Healthy lifestyle, including a balanced diet, in combination with regular aerobic exercise and low-weight resistance training, are important to maintain good glycaemic control.11

\section{Treating patients with diabetes and COVID-19 infection}

In a patient with diabetes and a confirmed COVID-19 infection, strict blood glucose monitoring is required, both in the hospital and quarantine environment. ${ }^{19}$ In most cases of mild COVID-19 infection, antidiabetic treatment should be followed as usual, with the exception of sodium-glucose cotransporter-2 (SGLT2) inhibitors, which may increase the risk of dehydration and diabetic ketoacidosis, demanding careful renal function monitoring. Interestingly, a study has been recently initiated to investigate a possible beneficial effect of dapagliflozin in hospitalised patients with COVID-19-related respiratory failure, and type 2 diabetes, cardiovascular disease and/or chronic kidney disease (DARE-19; ClinicalTrials.gov identifier: NCT04350593). ${ }^{20}$ Metformin is correlated with lactic acidosis, thus, in moderate or severe disease, discontinuation is suggested during hospitalisation. ${ }^{21}$ Sulfonylurea doses should be adjusted depending on patients' blood glucose levels, reduced food intake and the risk of hypoglycaemia, while discontinuation is definitely suggested in hospitalised patients. ${ }^{9,1,21}$ Although dipeptidyl peptidase 4 (DPP-4) inhibitors have been linked with upper respiratory infection risk, they do not alter the risk of pneumonia and there is not adequate evidence for or against their discontinuation.22

Although insulin usage in patients with diabetes infected with COVID-19 is correlated with poor prognosis, insulin therapy seems to be the primary strategy for hospitalised patients. ${ }^{18}$ Hospitalised patients should discontinue all oral antidiabetic medication. ${ }^{18,21}$ Insulin doses may need to be adjusted, based on the individualised therapeutic plan. In patients who receive basal insulin, fast-acting insulin is used for acute hyperglycaemia correction; blood glucose fluctuation during insulin therapy prerequisites strict and frequent monitoring. ${ }^{21}$ Effective blood glucose control in hospitalised patients (glycaemic variability within 3.9-10.0 mmol/L) was associated with reduced mortality compared with poorly controlled patients, in a retrospective, multicentre study in China. ${ }^{23}$ In ICU-admitted patients with diabetes and COVID-19, the main challenges remain efficient blood glucose control and high insulin requirements. Continuous tube feeding, the frequent use of intravenous corticosteroids and the prescription of vitamin C or other medications may contribute to an increase of glucose variability. Hospitalists should select the more suitable individualised insulin regimen, depending on the therapeutic plan. ${ }^{24}$

Hydroxychloroquine use has been initiated in many countries as a prophylaxis against COVID-19 infection and has shown to lead to a reduction of viral load and glycated haemoglobin $(\mathrm{HDA} 1 \mathrm{C}) .{ }^{25}$ However, hydroxychloroquine may cause hypoglycaemia. In addition, the combination of hydroxychloroquine with metformin is potentially toxic. 25,26 Despite the benefits of hydroxychloroquine on good glycaemic control, long-term use in patients with diabetes is correlated with fatal cardiac arrhythmias and retinal toxicity. Serious ventricular arrhythmias may occur, especially in combination with drugs that prolong QT interval, such as macrolides, antiviral drugs and antihistamines; therefore, a careful risk-benefit assessment should be performed before the use of hydroxychloroquine in patients with diabetes and COVID-19. ${ }^{27}$

\section{Conclusion}

Diabetes and COVID-19 infection may lead the patient in a vicious cycle, with unpredictable and possibly unfavourable outcomes. Given that there is, so far, limited scientific evidence, most management directions for diabetes during the COVID-19 pandemic are coming from expert opinions around the world. For this reason, it is profoundly important that infection prevention measures should be strictly followed until an effective treatment is developed, hopefully in the near future. $\square$
1. World Health Organization. Naming the coronavirus disease (COVID-19) and the virus that causes it. 2020. Available at: www.who.int/emergencies/diseases/novel-coronavirus-2019/ technical-guidance/naming-the-coronavirus-disease-(covid2019)-and-the-virus-that-causes-it (accessed 10 June 2020).

2. Ge H, Wang X, Yuan $X$, et al. The epidemiology and clinical information about COVID-19. Eur J Clin Microbiol Infect Dis. 2020;1-9.

3. Mahase E. COVID-19: WHO declares pandemic because of "alarming levels" of spread, severity, and inaction. BMJ. 2020;368:m1036.

4. Yang CL, Qiu X, Zeng YK, et al. Coronavirus disease 2019: a clinical review. Eur Rev Med Pharmacol Sci. 2020:24:4585-96. Clinical review. Eur Revi for Systems Science and Engin-ering (CSSE) at Joh Hopk for Systems science and Engin-19 Dashboard by he Center University (JHU). Available at: https://coronavirus.jhu.edu/map. html (accessed 10 June 2020)

6. Dariya B, Nagaraju GP. Understanding novel COVID-19: its impact on organ failure and risk assessment for diabetic and cancer patients. Cytokine Growth Factor Rev. 2020;53:43-52.
7. Huang I, Lim MA, Pranata R. Diabetes mellitus is associated with increased mortality and severity of disease in COVID-19 pneumonia - a systematic review, meta-analysis, and meta-regression. Diabetes Metab Syndr. 2020;14:395-403.

8. Muniyappa R, Gubbi S. COVID-19 pandemic, coronaviruses, and diabetes mellitus. Am J Physiol Endocrinol Metab. 2020;318:E736-41.

9. Pal R, Bhadada SK. COVID-19 and diabetes mellitus: An unholy interaction of two pandemics. Diabetes Metab Syndr. 2020;14:513-7.

10. Wang X, Fang $X$, Cai Z, et al. Comorbid chronic diseases and acute organ injuries are strongly correlated with disease severity and mortality among COVID-19 patients: a systemic review and meta-analysis. Research (Wash D C). 2020;2020:2402961.

11. Singh AK, Gupta R, Ghosh A, Misra A. Diabetes in CoVID-19: Prevalence, pathophysiology, prognosis and practical Prevalence, pathophysiology, prognosis and practical
considerations. Diabetes Metab Syndr. 2020;14:303-10.

2. Hussain A, Bhowmik B, do Vale Moreira NC. COVID-19 and diabetes: knowledge in progress. Diabetes Res Clin Pract.
2020;162:108142

13. Khera R, Clark C, Lu Y, et al. Association of angiotensin-converting enzyme inhibitors and angiotensin receptor blockers with the risk of hospitalization and death in hypertensive patients with coronavirus disease-19. medRxiv 2020; doi:10.1101/2020.05.17.20104943 [Preprint].

14. Kosinski C, Zanchi A, Wojtusciszyn A. Diabetes and COVID-19 infection. Rev Med Suisse. 2020;16:939-43.

5. Wang W, Lu J, Gu W, et al. Care for diabetes with COVID-19: advice from China. J Diabetes. 2020;12:417-9.

16. Angelidi AM, Belanger MJ, Mantzoros CS. COVID-19 and diabetes mellitus: What we know, how our patients should be treated now, and what should happen next. Metabolism. 2020:107:154245.

17. European Society of Cardiology. Position Statement of the ESC Council on Hypertension on ACE-Inhibitors and Angiotensin Council on Hypertension on ACE-Inhibitors and Angiotensi
Receptor Blockers. 2020. Available at: www.escardio.org/ Councils/Council-on-Hypertension-(CHT)/News/positionstatement-of-the-esc-council-on-hypertension-on-aceinhibitors-and-ang (accessed 10 June 2020). 
18. Chen Y, Yang D, Cheng B, et al. Clinical characteristics and outcomes of patients with diabetes and COVID-19 in association with glucose-lowering medication. Diabetes Care. 2020;43:1399-407.

19. Rhee EJ, Kim JH, Moon SJ, Lee WY. Encountering COVID-19 as endocrinologists. Endocrinol Metab. 2020;35:197-205.

20. Tucker ME. New study of diabetes drug for COVID-19 raises eyebrows. 2020. Available at: www.medscape.com/ viewarticle/929716 (accessed 10 June 2020).

21. Bornstein SR, Rubino F, Khunti K, et al. Practical recommendations for the management of diabetes in patients with COVID-19. Lancet Diabetes Endocrinol. 2020;8:546-50.
22. Iacobellis G. COVID-19 and diabetes: can DPP4 inhibition play a role? Diabetes Res Clin Pract 2020:162:108125.

23. Zhu L, She Z-G, Cheng X, et al. Association of blood glucose control and outcomes in patients with COVID-19 and pre-existing type 2 diabetes. Cell Metab. 2020:31:1068-77.

24. Hamdy O, Gabbay RA. Early observation and mitigation of challenges in diabetes management of COVID-19 patients in challenges in diabetes management of COVID-19
critical care units. Diabetes Care. 2020;43:e81-2.

25. Singh AK, Singh A, Shaikh A, et al. Chloroquine and

hydroxychloroquine in the treatment of COVID-19 with or

without diabetes: a systematic search and a narrative review with a special reference to India and other developing countries. Diabetes Metab Syndr. 2020;14:241-6.

26. Rajeshkumar NV, Yabuuchi S, Pai SG, et al. Fatal toxicity of chloroquine or hydroxychloroquine with metformin in mice. bioRxiv. 2020; 018556; doi:10.1101/2020.03.31.018556 [Preprint].

27. Meissner ME. Considerations for hydroxychloroquine use in patients with diabetes. 2020. Available at:

patients with diabetes. 2020. Available at:
www.endocrinologyadvisor.com/home/topics/diabetes/ www.endocrinologyadvisor.com/home/topics/diabetes/
hydroxychloroquine-antihyperglycemic-effects-in-diabeteshydroxychloroquine-antihyperglycemic
and-covid-19/ (accessed 10 June 2020). 